\title{
Glasses-Free 3D Display System Using Grating Film for Viewing Angle Control
}

\author{
Masahide Kuwata and Kunio Sakamoto \\ Department of Intelligence and Informatics, Konan University \\ 8-9-1 Okamoto, Higashinada, Kobe 658-8501, Japan \\ kunio@konan-u.ac.jp
}

\begin{abstract}
We developed a glasses-free 3D stereoscopic display using an LCD display panel and a special grating film for stereoscopic viewing. The display screen is divided in half in order that left and right regions provide the stereoscopic images for left and right eyes. Because both stereoscopic images are not in the same position, it is difficult for the observer to view the $3 \mathrm{D}$ image by the stereoviewing. The grating film can solve this problem because it shifts both left and right images to the same position. Moreover this grating film can give us glasses-free 3D viewing because of its view control effect. As the result, the each eye can perceive separated stereoscopic images for left and right eyes without special glasses such as polarized glasses.
\end{abstract}

Keywords: optical film sheet, grating film, viewing angle control, stereoscopic 3D imaging, display.

\section{Introduction}

Conventional 3D movie systems with the special glasses such as polarized glasses provide us touchable spatial images. However, these 3D imaging systems are very expensive and large scale equipment. Our research group would like to realize the simple 3D imaging system to construct an interactive spatial imaging environment. The authors have researched the 3D displays and applications. We have ever proposed 3D displays using the slit as a parallax barrier, the lenticular screen and the holographic optical elements(HOEs) for displaying active image. This paper describes the 3D display using an optical film, which is sold at D.I.Y. stores, for easy 3D image viewing.

\section{Superimpose Stereoscopic Images}

To overlap left and right images, the authors use an optical film. This optical sheet is a flexible film with prisms designed to transport and diffuse the light. This sheet has interesting characteristics as follows; the prismatic phenomenon is observed and the doubling can be visible through the sheet like the Calcite. This doubling phenomenon occurs because the prism sheet diffracts two beams. These beams are called as the first order diffracted beam and the second order diffracted beam. Fig. 1 shows the doubling phenomenon. This interesting thing reminds us of method to superimpose left and right stereoscopic images. A grating diffracts or scatters a light beam with a designed angle. Using the doubling phenomenon, the authors shift the images for 
superimposing stereoscopic images by adjusting the interval between an optical sheet and image plane as shown in fig. 2. The optical grating film shifts both left and right images to the same position. The observer watches overlapped stereoscopic images for left and right eyes. This optical grating film is thin and works as a prism. The optical film appropriately locates apart from the display panel so that stereoscopic left and right images are overlapped. As shown in fig. 2(b), the left and right images are overlapped on the same plane. If these overlapped images can be separated into appropriate eyes, you can perceive the left image only by a left eye and the right image by a right eye.

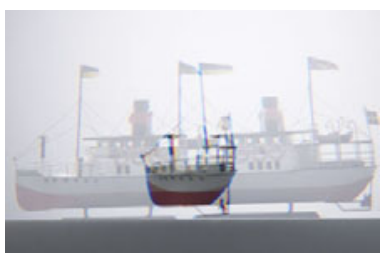

Fig. 1. Doubling phenomenon

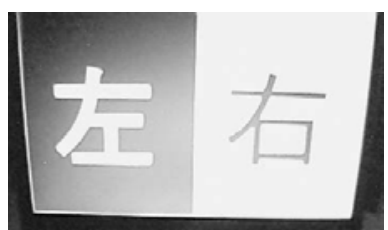

(a) side-by-side image

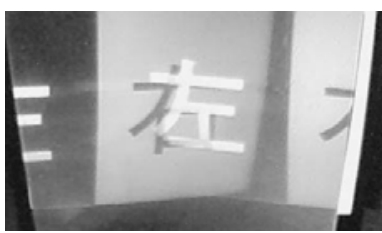

(b) view thru grating film

Fig. 2. Superimposing left and right images

\section{View Control Effect of Grating Film}

The authors firstly used the "SOLF" ${ }^{\mathrm{TM}}$ " optical film for overlapping left and right image as shown in fig. 2. But you need to wear polarized glasses for separating stereo image. To deliver left and right images into appropriate eyes though you wear no glasses, we use a new grating film with view control effect. The author unexpectedly found this sheet in D.I.Y. materials floor at Sannomiya Store of Tokyu Hands. This product name is unknown. We regard this sheet as a cheaper copy product of the SOLF sheet. However this sheet has a useful characteristic. Using this film, you can see through the film from the left, but not from the right as shown in fig 3 . One of the miraculous features is that it can be either transparent or grating sheet, so that it looks either like transparent glass or prism, depending on the angle of sight.

It is useful characteristics for 3D viewing that you can control what can and what cannot be seen depending on which side the viewer is on, or what angle the viewer is looking from. Using the miracle of this visibility control as shown in fig 3 , it enables us to perceive left images by the only left eye and right images by the only right eye. As shown in fig 4, the view control film passes the light within a designed angle. Let's design the optical layout assuming that 9-inch display panel is used. The width of the 9 -inch panel is approximately $200 \mathrm{~mm}$. As shown in the fig. 4 , the ray of a left image is emitted with an angle $\alpha$ to vertical and it reaches into the left eye after the ray is diffracted by an optical grating film. Meanwhile the grating film doesn't diffract the ray with an angle $\beta$, which passes into the right eye. Therefore the left image is observed by the only left eye because the ray with an angle $\beta$ to vertical is blocked by the optical film. The rays of a right image are the same as the left image. Using this grating film, the observer can perceive the left image only by a left eye and the right image by a right eye with no glasses because the film with view control effect restricts the direction of scattering light after the grating film overlays left and right images at the same position. Therefore, the observers, who wear no glasses, can view the $3 \mathrm{D}$ images by the binocular viewing. 
We have developed the prototype glasses-free stereoscopic 3D display using two commercial LCD panels for playing 3D contents by portable DVD players. The size of panels is 7.24-inch and its width of viewscreen is $103 \mathrm{~mm}$. Assuming the 9-inch panel, the interval between the center of viewscreen is $100 \mathrm{~mm}$. Both panels are side by-side and each LCD displays a left image for the left eye and a right image for the right eye. The grating film plate is positioned $92 \mathrm{~mm}$ apart from the display panels so that stereoscopic left and right images are overlapped. The observer perceives an overlapped image through the grating film within a viewing window as shown in fig. 5. In trial display, the observation distance is $400 \mathrm{~mm}$. Because of overlapping images by optical film, the observer can see the 3D image at approximately $300 \mathrm{~mm}$ apart from the viewing window of a display box. Since viewing positions of left and right images are restricted, the observers can view the 3D images by the binocular stereo viewing without special glasses.

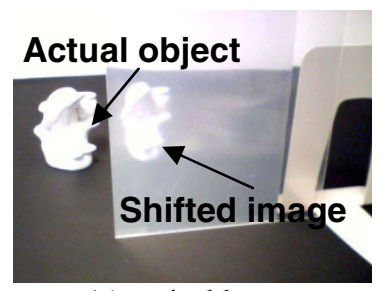

(a) optical layout

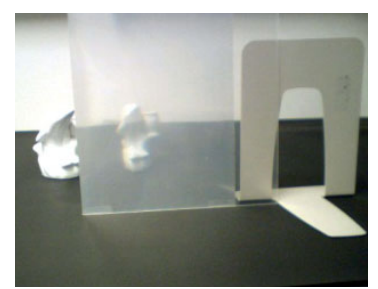

(b) viewing from left

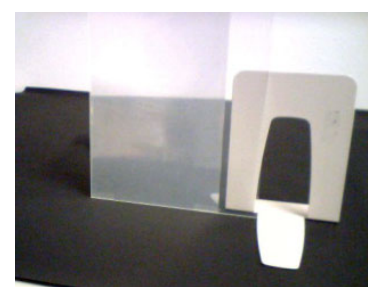

(c) viewing from right

Fig. 3. View control effect of grating film

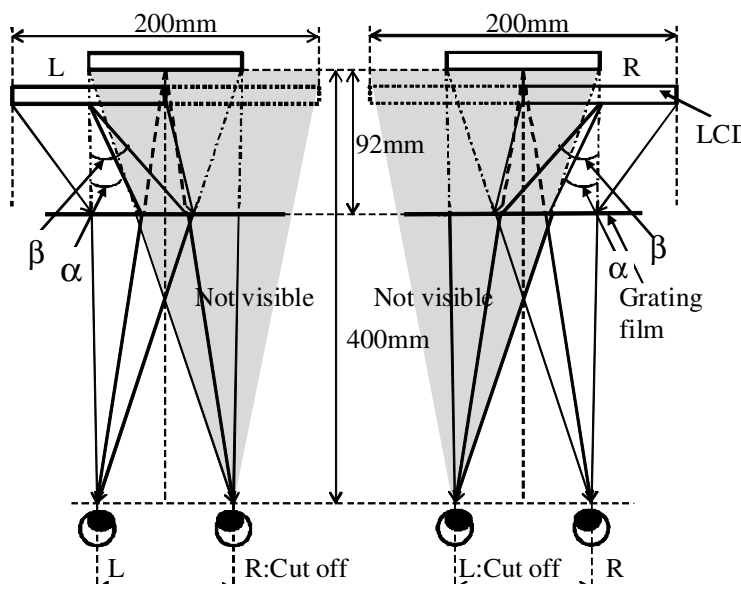

Fig. 4. Optical design for $3 \mathrm{D}$ viewing

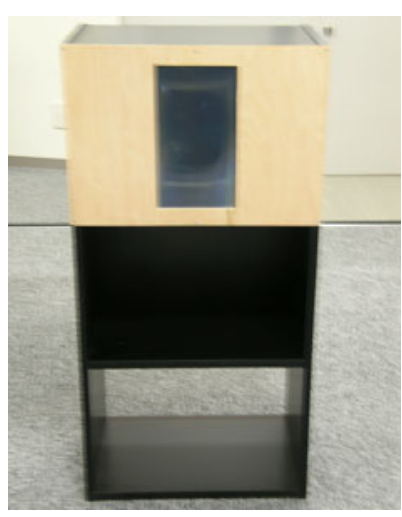

Fig. 5. Appearance of 3D display (KNA-30)

Acknowledgments. This research is partially supported by "Grant-in-Aid for Young Scientists(B)" \#20700112 and "Scientific Research (C) (General)" \#20500481 from Ministry of Education, Culture, Sports, Science and Technology Japan(MEXT). 